\title{
The bacterial profile of neonatal sepsis and antibiotic use in the tertiary care NICU of Kosovo
}

\begin{abstract}
Neonatal infections including sepsis, pneumonia, tetanus, and diarrhea are among the leading causes of neonatal mortality worldwide. We performed a retrospective analysis of neonatal infections referred to the only tertiary referral Neonatal Intensive Care Unit (NICU) in Kosovo from January 1, 2013 to December 31, 2014. Of 1426 admissions, $702(49.2 \%)$ were diagnosed with an infection. Proven or probable sepsis occurred in 107 patients, with $63.6 \%$ classified as early onset. Early onset sepsis cases were treated with an average of 4.37 antibiotics with $94.3 \%$ receiving vancomycin and $88.57 \%$ receiving carbapenem. No antibiotic sensitivity data was available at the time of data collection and the reasons for these prescribing practices which clearly deviate from standards in the United States are unclear. In conclusion, neonate sepsis rates continue to be high NICU patients in Kosovo. Further investigations and quality improvement initiatives need to be done to address antibiotic resistance patterns and antibiotic stewardship.
\end{abstract}

Keywords: intensive care units, neonatal, anti-bacterial agents, perinatal mortality, kosovo, neonatal sepsis
Volume 8 Issue 2 - 2018

\author{
Julia Segal,' Mynevere Hoxha, M.D. ${ }^{2}$ Simone \\ Wien, ${ }^{3}$ llir Hoxha, Ph.D., M.D., M.Sc. ${ }^{4}$ \\ Rebecca T Emeny, Ph.D., MPH ${ }^{5}$ Alka Dev, \\ DrPH ${ }^{6}$ Tyler Hartman, M.D. ${ }^{7}$ \\ 'Geisel School of Medicine at Dartmouth, United States \\ ${ }^{2}$ Neonatology, University Clinical Center of Kosova, Kosovo \\ ${ }^{3}$ Department of Geography, Dartmouth College, USA \\ ${ }^{4}$ Department of Community and Family Medicine, Geisel School \\ of Medicine at Dartmouth, USA \\ Department of Health Management, Heimerer College, USA \\ ${ }^{5}$ Research Design and Epidemiology Core, Dartmouth \\ SYNERGY,The Dartmouth Institute for Health Policy and \\ Clinical Practice, United States \\ International Health, Dartmouth-Hitchcock Medical Center, \\ United States \\ ${ }^{7}$ Neonatology, International Health, Dartmouth-Hitchcock \\ Medical Center, United States
}

Correspondence: Julia Segal, Geisel School of Medicine at Dartmouth, USA, Email JuliaEllenSegal@gmail.com

Received:September 14,20I7 | Published: April II, 20 I8
Abbreviations: UCCK: University Clinical Center of Kosovo; NICU: Neonatal intensive care unit

\section{Introduction}

Reducing neonatal death is critical for lowering child mortality. In $2013,45 \%$ of deaths under the age of 5 years worldwide occurred during the first month of life, with $35 \%$ of deaths occurring in the first seven days after birth. ${ }^{1}$ Alarmingly, there were nearly as many estimated stillbirths as there were neonatal deaths indicating a severe gap in Perinatal care. Neonatal infections including sepsis, pneumonia, tetanus, and diarrhea are among the leading causes of neonatal mortality worldwide. ${ }^{2}$ Severe infections accounted for $24 \%$ of all neonatal deaths. ${ }^{3}$ globally, neonatal sepsis in particular remains a leading cause of neonatal death. ${ }^{2}$ The Republic of Kosovo is a middle-income country in the central Balkan Peninsula. Following the 1999 NATO air strikes on former Yugoslavia, Kosovo entered a post-conflict transition focused on infrastructure, economic growth, and establishment of organized healthcare. Although the Perinatal mortality rate in Kosovo has declined from 29.1 per 1000 births in 2000 to 12.1 per 1000 births in 2015 , it remains one of the highest in Europe. ${ }^{4}$ Low investments in healthcare spending, inadequate number of healthcare professionals, and infection control concerns have prevented faster reductions in the neonatal mortality rate, thus contributing to Kosovo's failure to meet Millennium Development Goal 4 to reduce child mortality by two-thirds by $2015 .{ }^{5}$ Regional data on infection rates, common bacterial pathogens, and antibiotic resistance are essential to develop strategies to reduce neonatal mortality. However, national data surrounding infection rates and pathogens in Kosovo remain limited. In 2011, total antibiotic use in Kosovo was among the highest in Eastern Europe at just over 25 defined daily doses per 1000 inhabitants per day, raising concern about the development of antibiotic resistance. ${ }^{6}$ Evaluation of antibiotic use is essential to develop strategies to further lower the neonatal mortality rate in Kosovo, determine responsible antibiotic use, and limit antibiotic resistance patterns. The aim of this study was to review neonatal infections, in particular early onset and late onset sepsis, and also to characterize athogens and antibiotic use among patients referred to the University Clinical Center of Kosovo (UCCK), Kosovo's only level III neonatal intensive care unit (NICU) referral center.

\section{Materials and method}

A retrospective analysis of neonates referred to UCCK from January 1, 2013 to December 31, 2014 was performed. UCCK is the only tertiary referral Neonatal Intensive Care Unit (NICU) in Kosovo and contains 180 beds, 50 of which are designed for neonatal critical care. Twenty-five neonatologists or pediatricians and ninety-one nurses staff the unit. We identified patients who met the inclusion criteria of being referred and admitted to the UCCK NICU during 2013 or 2014 with sepsis or other clinical diagnosis of infection. Medical records of NICU patients referred and admitted to UCCK were reviewed and data was extracted on the clinical and laboratory indicators regarding infection. Data analysis was performed by four independent outside reviewers. At intermittent intervals, random patient records were reviewed independently by this research team in order to ensure the validity of data collection. If data was inconsistent, the case was excluded from analysis. Demographic data included birth weight, gestational age, postnatal age, and treatment as well as 
survival outcome. Clinical and laboratory data included the number of days between birth and admission to the NICU, admitting and discharge diagnoses, and pharmacological treatment. Neonates were classified as having proven sepsis, probable sepsis, or other infection. We defined proven sepsis based on clinical signs, laboratory findings, and confirmation by blood culture. A positive blood culture was considered growth within 5 days on BacT/ALERT PF culture medium using minimum of $0.5 \mathrm{cc}$ of blood with 2 cultures performed if possible. Patients with clinical symptoms and/or laboratory findings with no growth on culture medium were considered to have probable sepsis. Blood cultures positive for Coagulase-negative staphylococci were considered contaminants due to a lack of central line use in the UCCK NICU. Other infections were defined by clinical diagnosis. Culture -positive sepsis was categorized as early onset sepsis, defined as 0-6 days, and late onset sepsis, defined as 7 days-3 months. Only patient data from those admitted in 2014 included pharmacological treatment information.

\section{Results}

There were 1426 neonates referred to the NICU between 2013 and 2014 and $702(49.2 \%)$ were diagnosed with any infection (Figure 1). Of total admissions, $18.9 \%$ were diagnosed with proven sepsis or probable sepsis. Out of the 107 proven sepsis cases, 68 cases $(63.6 \%)$ were early onset and 37 cases $(34.6 \%)$ were late onset. There was no data regarding onset of infection in $1.9 \%$ of neonates. Of probable sepsis cases, 75 cases grew Coagulase-negative staphylococcus, which was assumed to be a contaminant by convention. Various demographics of neonates in our sample are summarized here. Of 1426 referrals to UCCK during 2013-2014 periods, 1331 were discharged and 85 expired. Of these referrals, $60.5 \%$ were male, $25.6 \%$ were born by $\mathrm{C}$-section, and the most common diagnosis was "other infection" which included acute bronchiolitis, bronchopneumonia, and pneumonia. A majority of neonates were born at or near term; $16.4 \%$ were born premature. In addition, $82.9 \%$ of neonates were born weighing between 2.00 and $3.99 \mathrm{~kg}, 10.7 \%$ were born above $4.0 \mathrm{~kg}$, and $5.6 \%$ weighed between $1.00-1.99 \mathrm{~kg}$. Almost half $(\mathrm{n}=332$, $44.6 \%$ ) of the 745 total patients admitted during 2014 were treated with vancomycin during their stay, and $32.6 \%$ were treated with a carbapenem (Figure 2). Neonates with a gestational age under 37 weeks were treated with an average of 3.24 antibiotics, while neonates with a gestational age between 37 and 42 weeks were treated with an average of 1.93 antibiotics. Table 1 demonstrates that in cases of proven sepsis there was a high bacterial prevalence of Klebsiella at $25.97 \%$, Enterobacter at $11.69 \%$ and Acinetobacter at $11.69 \%$ notably with no cases of group B streptococcus reported. Klebsiella species also comprised $18.18 \%$ of the bacterial profile for proven late onset sepsis with Enterobacter comprising $13.64 \%$ and Staphylococcus aureus $11.36 \%$ of the total cases.

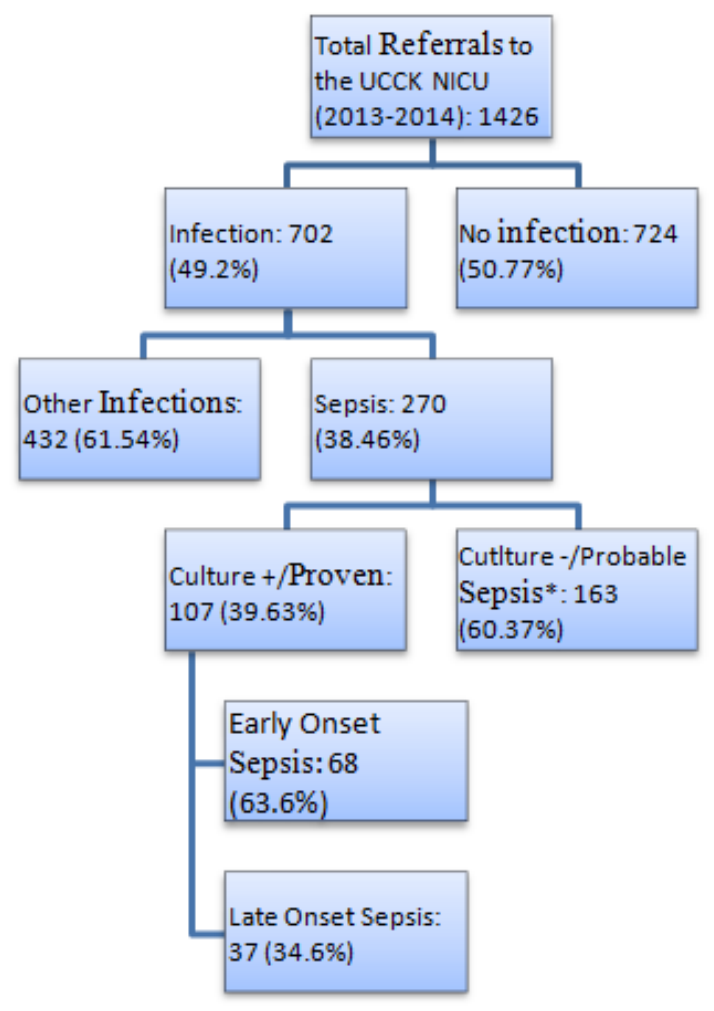

Figure I Referrals to the UCCK NICU (20|3-20|4).

Table I Frequency of pathogen infections in early and late sepsis

\begin{tabular}{llllllll}
\hline \multicolumn{2}{l}{ Proven Sepsis $(\mathbf{n}=\mathbf{I} \mathbf{2})$} & & & & \\
\hline & Early $(\mathbf{n}=\mathbf{7 7})$ & $\%$ & Late $(\mathbf{n}=\mathbf{4 4})$ & $\%$ & Total & $\%$ & Prob \\
\hline Not specified & 9 & 11.69 & 7 & 15.91 & 16 & 13.01 & 0.5097 \\
Coagulase-negative staphylococci & - & - & - & - & - & - & - \\
Klebsiella & 20 & 25.97 & 8 & 18.18 & 29 & 23.58 & 0.3282 \\
Serratia marcescens & 0 & 0 & 4 & 9.09 & 4 & 3.25 & 0.016 \\
Staph aureus & 8 & 10.39 & 5 & 11.36 & 14 & 11.38 & 0.8678 \\
Acinetobacter & 9 & 11.69 & 3 & 6.82 & 12 & 9.76 & 0.5328 \\
Achromobacter & 0 & 0 & 2 & 4.55 & 2 & 1.63 & 0.1303 \\
Enterobacter & 9 & 11.69 & 6 & 13.64 & 15 & 12.2 & 0.7544 \\
Enterococci & 7 & 9.09 & 0 & 0 & 7 & 5.69 & 0.0473 \\
Pseudomonas & 5 & 6.49 & 4 & 9.09 & 9 & 7.32 & 0.722 \\
E. coli & 1 & 1.3 & 3 & 6.82 & 4 & 3.25 & 0.136 \\
\hline Other & 9 & 11.69 & 2 & 4.55 & 11 & 8.94 & 0.3244 \\
\hline
\end{tabular}


Patients admitted in 2014 with early onset sepsis were treated with an average of 4.37 antibiotics, with $94.3 \%$ receiving vancomycin, $88.57 \%$ receiving a carbapenem, and $54.29 \%$ receiving cefipime. Of these patients, $14.04 \%$ were found to have been treated with a combination of vancomycin and imipenem. In addition, $8.77 \%$ of patients with early onset sepsis were treated with a combination of vancomycin, amikacin, imipenem, ampicillin, and cefipime, and $7.02 \%$ of patients were treated with a similar combination of vancomycin, gentamycin, imipenem, ampicillin, and cefipime. Patients with late onset sepsis were treated with an average of 3.24 antibiotics, with $82.35 \%$ receiving vancomycin, $82.35 \%$ receiving a carbapenem, and $88.24 \%$ receiving a $3^{\text {rd }}$ or $4^{\text {th }}$ generation Cephalosporin. Of these patients, $22.22 \%$ were found to have been treated with a combination of vancomycin and imipenem. Regardless of stage of sepsis, patients with any infection were administered an average of 2.39 antibiotics during their stay in the NICU. Patients with meningitis were treated with the greatest number of antibiotics, averaging 5.5 antibiotics per patient followed by patient with sepsis (Table 2). One patient with meningitis was treated with ten antibiotics, and another was treated with seven. No correlation was found between the rate of sepsis or infection with birth weight or gestational age.

Table 2 Average Number of Antibiotics per Case by Medical Condition (2014 data only)

\begin{tabular}{|c|c|c|c|c|c|c|c|}
\hline \multirow[b]{2}{*}{ Medical Condition } & \multicolumn{3}{|c|}{ (Early onset, 0-6 days) } & \multicolumn{4}{|c|}{ (Late onset, $>/=7$ days) } \\
\hline & $\mathbf{n}$ & $\begin{array}{l}\text { Mean Number of } \\
\text { Antibiotics per } \\
\text { Case }\end{array}$ & $\begin{array}{l}\text { Standard } \\
\text { Deviation }\end{array}$ & $\mathbf{n}$ & $\begin{array}{l}\text { Mean Number of } \\
\text { Antibiotics per } \\
\text { Case }\end{array}$ & $\begin{array}{l}\text { Standard } \\
\text { Deviation }\end{array}$ & $P$ values \\
\hline Meningitis & 3 & 7.33 & 2.52 & 9 & 4.89 & $\mathrm{I} .54$ & 0.0659 \\
\hline $\begin{array}{l}\text { Pneumonia, Acute } \\
\text { Bronchiolitis, } \\
\text { Bronchopneumonia }\end{array}$ & 6 & 2.83 & 1.72 & 127 & 1.45 & 1.17 & 0.0062 \\
\hline Sepsis & 57 & 4.37 & 2.17 & 36 & 3.24 & 1.37 & $<.0001$ \\
\hline Lymphadenitis & 2 & 3.5 & 0.71 & I & 3 & - & 0.6667 \\
\hline UTI & 4 & 3 & $1.4 \mathrm{I}$ & 7 & 2.14 & 0.9 & 0.2445 \\
\hline Phlegmon in femoral region & & $\mathrm{n} / \mathrm{a}$ & & I & 2 & - & $<.0001$ \\
\hline Neonatal mastitis & I & 4 & - & 3 & 1 & - & \\
\hline Abscess & & $\mathrm{n} / \mathrm{a}$ & & 5 & 1.4 & 0.55 & \\
\hline Staphylodermia & 5 & 1.2 & 1.3 & 8 & 1.5 & 0.54 & 0.6458 \\
\hline Omphalitis & II & 1.18 & 0.4 & 4 & 1.25 & 1.26 & 0.9215 \\
\hline Total Infection & 122 & 3.47 & 2 & 169 & 1.63 & 1.28 & $<.0001$ \\
\hline
\end{tabular}

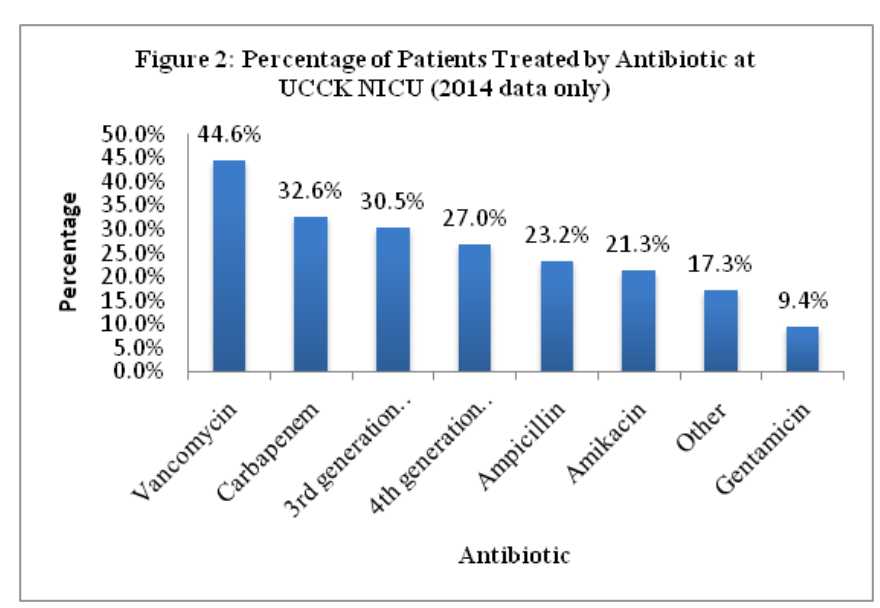

Figure 2 Percentage of Patients Treated by Antibiotic at UCCK NICU (20I4 data only).

\section{Discussion}

While neonatal deaths in the Europe have decreased substantially in the past decades, great disparity remains within the continent with the highest neonatal mortality rate estimated at 40 -fold the lowest. Compared to Western and Central Europe, Eastern Europe regularly has higher mortality and disability-adjusted life years across neonatal conditions, particularly neonatal sepsis. ${ }^{7}$ Kosovo continues to have one of the highest neonatal mortality rates in Europe and excessive empiric broad spectrum antibiotic use continues to be a major concern contributing to antibiotic resistance. Many studies have shown that the epidemiology of neonatal sepsis have both temporal and geographic variation, supporting the need for continuous monitoring of the organism causing neonatal sepsis in order to guide empiric antibiotic treatment. Our study is the first to report the bacterial etiology of both late and early onset sepsis in the only referral NICU in Kosovo. In our study, gram negative bacteria, particularly Klebsiella, were predominantly responsible for both early onset and late onset neonatal sepsis.

This is similar to a study by Zaidi et al. ${ }^{8}$ that reviewed over 11,000 infections and found that Klebsiella pneumoniae, other gram-negative rods (Escherichia coli, Pseudomonas spp, Acinetobacter spp), and Staphylococcus aureus were the major pathogens among 11,471 bloodstream isolates, suggesting that many of these sepsis cases may in fact be acquired from the hospital or community environment. ${ }^{8}$ Group B streptococcus has been reported to have a much higher incidence in high income countries than in low income countries, 
$73 \%$ vs $5 \%$ respectively, and there were no cases of either early onset sepsis or late onset sepsis with group B streptococcus in this study. This suggests that the etiology of neonatal sepsis in Kosovo is more similar to that found in developing countries than in developed countries, including its European counterparts where the incidence of infection was $36 \%$ in infants less than 3 months of age.

The high rates of Klebsiella noted at UCCK may also be due to an increase in highly resistant isolates in nearby regions. In Turkey, carbapenemase-producing Klebsiella pneumoniae isolates have been isolated from blood cultures of hospitalized patients. ${ }^{9}$ This study supports other neonatal studies that have shown that Klebsiella pathogens continue to have a high infection burden in low- and middleincome countries, the impact of which is exacerbated by antimicrobial resistance patterns. ${ }^{10} \mathrm{~A}$ recent study found that antimicrobial treatment guidelines are absent in many pediatric hospitals in Europe. Wide variation was found in existing guidelines concerning antimicrobial choice and duration of therapy, with twenty different combinations of antimicrobials documented for the treatment of neonatal sepsis. ${ }^{11}$ According to the $\mathrm{WHO}^{12}$ guide, Managing Newborn Problems, onset neonatal sepsis should be treated empirically with ampicillin and an amino glycoside, with cefotaxime replacing ampicillin if meningitis caused by a gram-negative organism is suspected (Organization, 2003). In terms of late onset neonatal sepsis, vancomycin is indicated if Coagulase-negative staphylococci is suspected, or a penicillin derivative such as oxacilin or nafcillin (or cefazolin as an alternative) if the organism is sensitive. ${ }^{13}$ The Pediatric Prevention Network, a collaborative effort of the CDC and the National Association of Children's Hospitals and Related Institutions, in general indicates NICUs and PICUs as high priority sites for the improvement of vancomycin use. Obtaining multiple blood cultures during sepsis evaluation in addition to implementing vancomycin restriction policies could help to curb vancomycin use and thus restrict the development of resistance. ${ }^{14}$ Further studies need to be done to appropriately tailor antibiotic regiments to treat pathogens while decreasing antibiotic exposure and the risk of resistance. These alterations could potentially lead to improved outcomes for patients and decreased cost.

\section{Acknowledgment}

Dartmouth Hitchcock-Medical Center, Department of Neonatology, University Clinical Care Centers of Kosovo, Action for Mothers and Children, H. Gilbert Welch, Geisel School of Medicine, for advising some areas of analysis. Mrika Aliu and Dafina Haliti of Action for Mothers and Children for national delivery data.

Research reported in this (publication/press release) was supported by the Dartmouth Clinical and Translational Science Institute, under award number UL1TR001086 from the National Center for Advancing Translational Sciences (NCATS) of the National Institutes of Health (NIH). The content is solely the responsibility of the author(s) and does not necessarily represent the official views of the NIH.

\section{Conflict of interest}

None.

\section{References}

1. GBD 2015 Child Mortality Collaborators. Global, regional, national, and selected subnational levels of stillbirths, neonatal, infant, and under-5 mortality, 1980-2015: a systematic analysis for the Global Burden of Disease Study 2015. Lancet. 2016;388(10053);1725-1774.

2. Liu L, Oza S, Hogan D, et al. Global, regional, and national causes of child mortality in 2000-13, with projections to inform post-2015 priorities: an updated systematic analysis. The Lancet. 2015;385(9966);430-440.

3. Black RE, Morris SS, Bryce J. Where and why are 10 million children dying every year? The Lancet. 2003;361(9376):22262234.

4. Azemi M, Gashi S, Berisha M, etal. Rate and time trend of perinatal, infant, maternal mortality, natality and natural population growth in Kosovo. Mater Sociomed. 2012;24(4):238-241.

5. Pia Rebello Britto PJ. Joined Hands Better Childhood. Retrieved from Unicef: The Edward Zigler Center of Child Development and Social Policy, August 31, 2011;4-104.

6. Ann Versporten, Ganna Bolokhovets, Lilit Ghazaryan, et al. Antibiotic use in eastern Europe: a cross-national database study in coordination with the WHO Regional Office for Europe. The Lancet Infectious Diseases. 2014;14(5);381-387.

7. Setiawan C. Background Paper 6.23 Neonatal Conditions. Priority Medicines for Europe and the World A Public Health Approach to Innovation. 2013;1-50.

8. Zaidi AK, Thaver D, Ali SA, et al. Pathogens associated with sepsis in newborns and young infants in developing countries. Pediatr Infect Dis J. 2009;28(1):S10-S18.

9. Koksal Cakirlar, N Gonullu, F Kalayci. Detection of carbapenemase genes OXA-48, VIM, IMP, KPC and NDM in carbapenemaseproducing klebsiella pneumoniae isolates from blood cultures of hospitalized patients in Istanbul, Turkey. International Journal of Infectious Diseases. 2016;45(1);99.

10. Waters D, Jawad I, et al. Ahmad A. Aetiology of communityacquired neonatal sepsis in low and middle income countries. Journal of Global Health. 2011;1(2):154-170.

11. Spyridis N, Syridou G, Goossens H, et al. Variation in paediatric hospital antibiotic guidelines in Europe. Arch Dis Child. 2016;101(1);72-76.

12. World Health Organization (WHO). Managing newborn problems: a guide for doctors, nurses, and midwives. Sexual and reproductive health. 200;336.

13. Caserta MT. Neonatal sepsis. USA; 2015.

14. Keyserling HL, Sinkowitz-Cochran RL, Harris JM $2^{\text {nd }}$, et al. Vancomycin use in hospitalized pediatric patients. Pediatrics. 2003;112(2);e104-e111. 